eISSN: 2387-1555

DOI: http://dx.doi.org/10.14201/rea20209155164

\title{
EL CORRIMIENTO DE LA FRONTERA. UN MOMENTO EN LA CONFIGURACIÓN DE LAS SOCIEDADES DE RANCHEROS EN LOS ALTOS Y EI NORTE DE JALISCO.
}

The landslide of the frontier. A period in the configuration of rancher's societies in Los Altos and the North of Jalisco

Deslocamento da fronteira. Um periodo na configuração das sociedades de fazendeiros em Los Altos e no norte de Jalisco

\author{
Andrés FÁBREGAS PUIG \\ Centro de Investigaciones y Estudios Superiores en Antropologia Social. Unidad Occidente \\ $\triangle$ afabregas@ciesas.edu.mx
}

Fecha de recepción: 18 de septiembre de 2019

Fecha de aceptación: 9 de diciembre de 2019

Resumen: La concentración de los antropólogos mexicanos en las cuestiones planteadas por el indigenismo, ocasionó el descuido del estudio de las sociedades de rancheros. Tal situación era inadmisible más, si se tiene en cuenta que de las sociedades rancheras del Occidente de México provienen los simbolismos culturales nacionales de México: el tequila, la charrería, el charro. En este texto, se describe y reflexiona acerca de la formación de las sociedades rancheras en México y cómo influyó en ello la situación de frontera durante el período colonial, bajo el Virreynato de la Nueva España y la Audiencia de la Nueva Galicia.

Palabras claves: sociedad; rancheros; Jalisco fronteras; corrimiento.

Summary: The concentration of mexican anthopologists in the study of indians people, has a result the no study of rancher's societies. We do not admit this situation, because rancher's societies from the occident of Mexico, are the origin of cultural symbols of the nation: tequila, ranchers. In this text, we describe the configuration of the societies of ranchers and whtat was the role of the frontier during the colonial period in such configuration under the rule of New Spain and the Audience of New Galicia.

Keywords: societies; ranchers; frontiers; Jalisco; landslide.

Resumo: A concentração de antropólogos mexicanos nas questões levantadas pelos povos indígenas causou a negligência do estudo das sociedades de fazendeiros. Tal situação era ainda mais inadmissível, se considerarmos que as sociedades de fazenda do México ocidental provêm dos simbolismos culturais nacionais do México: tequila, charrería, charro. Neste texto, descrevemos e refletimos sobre a formação de sociedades de fazendas no México e como a situação da fronteira a influenciou durante o período colonial, sob o vice-reinado da Nova Espanha e o público da Nova Galiza.

Palavras-chave: sociedade; fazendeiros; fronteiras de Jalisco; delocamento.

\section{Introducción.}

Al iniciarse la década de los años 1970, la antropología en México se concentraba en el estudio de las culturas y las sociedades indígenas del país. Dicha concentración obedecía a los propósitos del Estado Nacional Mexicano de forjar una sociedad nacional con su respectiva cultura nacional, aplicando una política hacia los pueblos originarios que fue ampliamente conocida como indigenismo. El punto de partida era la convicción que, por cierto, viene desde por lo menos el siglo XVIII, en la imposibilidad de forjar una Nación y un 
Estado en un contexto de diversidad cultural como la presentada por México. Se consideraba que el mestizo, dicho en su más amplia acepción, era el protagonista de la historia mexicana y por lo tanto las culturas y sociedades originarias que conservaban sus modos de ser, debían sujetarse a un intenso y acelerado proceso de aculturación que resultaría en la asimilación de las mismas y su incorporación al mestizaje. El resultado final de esos procesos se confiaba que fuese una nación configurada alrededor de una cultura nacional, sostenida por una sociedad nacional que, a su vez, debía ser el soporte del Estado Nacional Mexicano. El antropólogo mexicano que con mayor claridad expuso este punto de vista, además de aplicarlos prácticamente, fue Gonzalo Aguirre Beltrán, autor de una vasta e importante obra, pionera en campos como el de la etnohistoria, los estudios de regiones interculturales o los primeros trabajos sobre la población de origen afrocaribeño en México. De esa vasta obra, y en términos de lo que he expuesto, sugiero a quien tenga interés en profundizar acerca del indigenismo, leer el libro autobiográfico de Gonzalo Aguirre Beltrán, El quehacery el pensar de la antropología en México (Puebla: BUAP, 1986).

En el contexto intelectual de la antropología en México que he expuesto con brevedad, resaltaba el prototipo de mexicano alentado por el Estado Nacional en estrecha vinculación con los antropólogos mismos. Ese prototipo colocaba a los rancheros de Jalisco como el mexicano promedio, como el ideal al que debería ajustarse la población mexicana. Los símbolos que acompañaban a ese prototipo eran la figura del charro, la charrería como deporte nacional, el tequila como la bebida nacional y el mariachi como la música nacional. En un momento dado, el cine mexicano se apoyó sobre estos simbolismos o incluso la paraestatal PEMEX adoptó al charro como el símbolo de la empresa. Interesante es observar que la antropología dio por hecho todo ello y no puso atención en estudiar cómo se forjaron las sociedades de rancheros en México y cómo llegaron a colocarse como símbolos de una sociedad nacional y su cultura.

En los inicios de los 1970, me encontraba estudiando antropología bajo la influencia de Guillermo Bonfil Batalla y Ángel Palerm, antropólogos muy diferentes entre sí. A Bonfil le interesaba crear conocimiento acerca de lo que él llamó el México Profundo (1990), título de su libro más divulgado, además de liderar la crítica al indigenismo y a la obra de Gonzalo Aguirre Beltrán. En cambio, Ángel Palerm había introducido temas nuevos a la antropología en México, como el del campesinado o la reflexión sobre el Estado y las sociedades nacionales, además de compartir la crítica al indigenismo, pero desde ángulos diferentes a los señalados por Bonfil. En mi caso, había pasado por la experiencia de trabajar junto a Bonfil en la región de Chalco-Amecameca y haber escrito sobre temas que aluden a la cosmovisión del orbe náhuatl de aquella región. En los años de 1971-1972, llevé a cabo una estancia doctoral en la Universidad de Nueva York en Stony Brook en donde estudié con Pedro Carrasco, Pedro Armillas y Phil Weigand, lo que amplió mi mirada acerca de la antropología en general y en particular en México.

A mediados del año de 1973, de regreso a México, sostuve varias conversaciones con Ángel Palerm quien había refundado el Departamento de Antropología de la Universidad Iberoamericana en la ciudad de México. En ese mismo año, Bonfil, Palerm y Aguirre Beltrán se unieron para fundar el Centro de Investigaciones y Estudios Superiores del Instituto Nacional de Antropología e Historia, cuyo primer Director fue el propio Ángel Palerm, mientras Bonfil fungía como Director General del INAH y Aguirre Beltrán era Sub-Secretario de Cultura y Director General del Instituto Nacional Indigenista (INI). En ese contexto, durante la conversación mencionada con Palerm, este señaló la importancia de llevar a cabo un estudio antropológico de los Altos de Jalisco no sólo porque no había en ese momento una visión regional de aquella parte del país, sino por la importancia simbólica que tenía a nivel nacional. Además, y en ello coincidí 
con Palerm, había que llevar a la antropología en México a temas y regiones que rompieran con el círculo recurrente de estudiar sólo a las poblaciones indígenas, sin negar la importancia que estas debían seguir manteniendo para los antropólogos y el país. Palerm, en compañía de su amigo Eric Wolf, habían recorrido los Altos de Jalisco y El Bajío Mexicano, impresionándose por las características de aquellas regiones, como lo escribió posteriormente el segundo en su conocido libro Pueblos y Culturas de Mesoamérica (México, editorial Era, 1979). Así que el tema estaba a la puerta: el estudio antropológico regional de los Altos de Jalisco.

Termino esta Introducción comentando que se formó un grupo de investigación que se puso bajo mi responsabilidad mientras el patrocinio del proyecto quedó en el marco institucional del CIS-INAH.

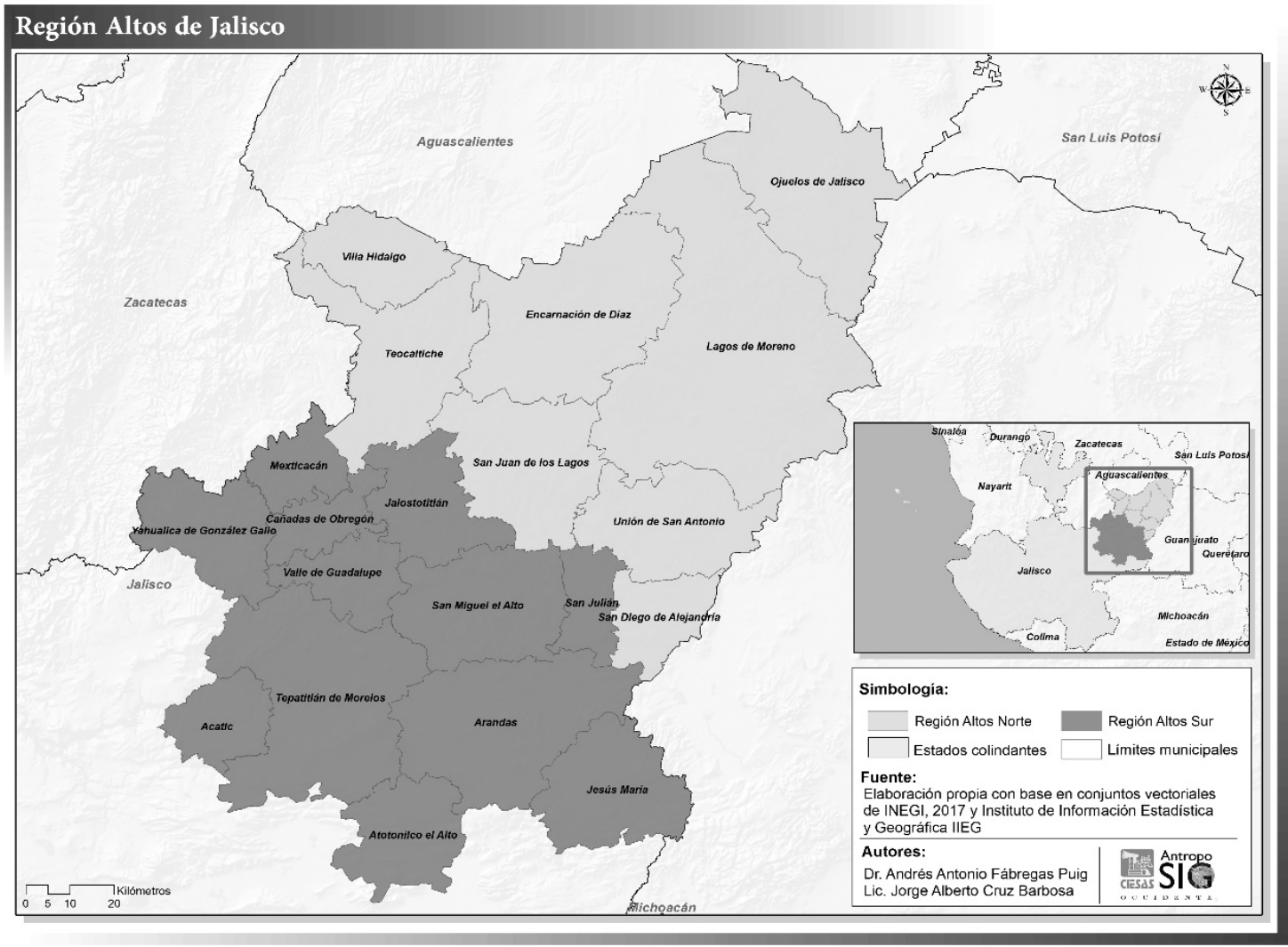

Figura n. ${ }^{\circ} 1$.

\section{Los términos conceptuales del estudio de los Altos de Jalisco}

En aquel año de 1973 se conocían en México los puntos de vista que Ángel Palerm introdujo a la antropología en el país, entre ellos, el método de la ecología-cultural. Retomé ese método en el estudio de los Altos de Jalisco. Introduje, además, el punto de vista de la teoría de la frontera, combinando a Frederick Jackson Turner y Owen Lattimore pero dentro de una orientación Histórica-Crítica, influenciado por la obra y la discusión con Lawrence Krader. Finalmente, con ese bagaje teórico, decidí seguir un acercamiento etnohistórico, retomando sugerencias de Gonzalo Aguirre Beltrán y de Luís González y González.

El desarrollo de los puntos de vista expuestos se inició con un recorrido por los Altos de Jalisco para identificar los límites regionales y las características del ámbito regional. Ello permitió, dentro de los parámetros del trabajo de campo de los antropólogos, los contactos con la gente y el paisaje. Facilitó también el decidir la distribución del grupo de investigación de tal manera que se abarcara a la región y su problemática. Importante fue el acceso a los archivos parroquiales, sobre todo, en las parroquias de Lagos de 
Moreno, Jalostotitlán y Tepatitlán. La exploración de los archivos se combinó con la etnografía, los resultados de que se disponían provenientes de la arqueología, la consulta de fuentes históricas y la tradición oral. Los resultados del proyecto han sido expuestos en una bibliografía variada escrita por los miembros del grupo que trabajó en la región alteña, además de un libro que escribí, La formación bistorica de una región. Los Altos de Jalisco (México, CIESAS, 1986).

\section{La visión antropológica de una región de rancheros}

Desde el análisis de los primeros datos históricos y etnohistóricos comparados con la etnografía, los Altos de Jalisco se manifiestan como un conjunto regional con sus límites bien establecidos y como una sociedad variada dentro de cierta homogeneidad cultural. Las características sobresalientes que en aquellos años presentaban los Altos de Jalisco fueron la fuente para elaborar la pregunta que sirvió de lanza a la pesquisa: ¿cómo se configuró este ámbito regional? La respuesta, armada en el proceso de investigación, arrojó las siguientes características:

a) El pasado de la región previo a la llegada de los castellanos, revela a un territorio usado por grupos nómadas que, sin desconocer la agricultura, la practicaron escasamente. El nomadismo y sus prácticas asociadas de caza y recolección, fue la estrategia más útil y eficaz para usar el territorio, dadas las características del mismo. En ese sentido, lo que hoy nombramos Altos de Jalisco, fue parte de la frontera ecológico-cultural entre el nomadismo y el sedentarismo, o si se prefiere, entre Mesoamérica y la Tierra Nómada.

b) Esa característica de Frontera se continúa bajo el contexto del régimen colonial. El territorio del que hablamos inicia su configuración regional bajo esas circunstancias, pero ahora, es la frontera como frente de expansión lo que marcará las características de la configuración regional. En efecto, los Altos de Jalisco es parte de la frontera de la expansión castellana hacia el norte, hacia lo profundo de la Gran Chichimeca, la Tierra Nómada, en el contexto conflictivo entre la Nueva España y la Nueva Galicia, ambas unidades administrativas con su propia Audiencia. Es aquí en donde cobra relevancia la colonización como una estrategia de expansión colonial en situaciones de frontera como la descrita. El enfrentamiento de la amplia alianza de los pueblos nómadas, genéricamente denominados chichimecas, hizo que las estrategias de colonización se modificaran por parte del Estado Español. En concreto, la llamada «Guerra del Mixton» (1540) obligó a imponer una estrategia de poblamiento operada por el Virrey Antonio de Mendoza primero y por la Audiencia de la Nueva Galicia después, que consistió en repartir tierras a pobladores a cambio de establecer y sostener la frontera contra los nómadas. Dicho todo ello a muy grandes rasgos. Pero fue en ese contexto que se introdujo la ganadería en lo que es hoy los Altos de Jalisco, un cambio ecológico-cultural radical, además del surgimiento de los ranchos como las unidades de producción básicas, además de los asentamientos de grupos de parientes que fueron reproduciendo la organización social de sus lugares de origen: el campo charro de Salamanca y la Peña de Francia, en Castilla, España. Fue esta estrategia de colonización la que resultó en una sociedad de pequeños y medianos propietarios, sin que se ignore la importancia de haciendas como la de Ciénega de Mata o la Hacienda de La Llave. Pero la tierra alteña fue más bien de pequeña y mediana propiedad. En ese contexto surgieron los tratos como el de la medianía y los jornales, que aún persisten. Aunado a ello, el uso del caballo se generalizó hasta convertirse en un rasgo cultural significativo. Los rancheros alteños son «hombres de a caballo» apreciándose en mucho las 
habilidades de un buen jinete. La charrería surgió en ese contexto de rancheros ganaderos, al calor de los herraderos y en general, del manejo, incluso lúdico, del ganado.

c) Los pobladores transportados desde España, se transformaron en rancheros y configuraron cultural y socialmente a la región de los Altos de Jalisco. Estos pobladores eran cristianos convencidos a la vez de la tarea de difundir el cristianismo bajo la égida de la Iglesia Católica. El catolicismo profundo de los rancheros alteños hunde sus raíces en los orígenes de la configuración regional y se tejió en la práctica de la guerra contra los chichimecas. Ello explica el papel preponderante de la Iglesia Católica como institución y la importancia del catolicismo aún en una actualidad en la que la diversidad religiosa está también presente.

d) En distintos momentos a lo largo de la configuración histórica de los Altos de Jalisco, los rancheros fueron acompañados en su hábitat por grupos indígenas de Tlaxcaltecas y aún Purépechas, además de núcleos de chichimecas cristianizados. Hubo también presencia de población afro, como lo prueba la documentación que informa de esclavos, de mercados de los mismos, de su uso como vaqueros en el manejo del ganado o en el servicio doméstico. Insistí en esta variedad de la sociedad desde la presentación de los primeros resultados de la investigación. En la actualidad esta variedad se ha profundizado incluso desde el punto de vista de la religión o la presencia de indígenas de Chiapas y de Oaxaca empleados en el trabajo del cultivo y jima del maguey tequilero.

e) Una importante característica cultural del ranchero en general en los Altos de Jalisco, en cierto sentido equiparable al del pionero del que habla Frederick Jackson Turner en su texto sobre el papel de la frontera en la historia americana, es su rechazo de la autoridad del Centralismo encarnado en el Virreynato y la defensa de sus decisiones locales. Es un punto muy interesante y que habría que discutir a la luz del presente etnográfico actual. Bastante de esta actitud explica, además de otros factores, el rechazo a la reforma agraria que el gobierno de Plutarco Elías Calles intentó operar desde el Estado Nacional. La llamada guerra cristera forma parte de esos mecanismos de rechazo de los que hablo, aunque no es solo ello lo que explica el estallido armado. Interviene también la forma del parentesco y las reglas de herencia, la operación de los linajes y la ideología del parentesco asociada a la identidad regional sellada por la religión. Complejo contexto que se seguirá discutiendo.

Después de los trabajos que abrieron la investigación antropológica regional en los Altos de Jalisco se han sucedido varias investigaciones, lo que es de celebrar. Ejemplo de ello son los trabajos de Guadalupe Rodríguez, José de Jesús Hernández López, Miguel Casillas, Alonso Hernández López, Celina Becerra, entre otros. Menciono incluso a los trabajos de comparación entre Los Altos de Jalisco y la Sierra de Ávila que hicimos en los años 1997-2000, el antropólogo español Pedro Tomé y yo, de cuyos resultados se publicaron varios libros, con el sello editorial del Colegio de Jalisco y la Institución Gran Duque de Alba, patrocinadoras de dicho proyecto. Así mismo, algunas de las afirmaciones que hice en mi libro sobre los Altos publicado en 1986 quedan atrás, aunque el modelo general de la configuración regional aún se sostiene. Además, existen en la actualidad antropólogos originarios de los Altos de Jalisco que llevan a cabo investigaciones en profundidad, actualizando el conocimiento de esta importante región mexicana. Los trabajos históricos, incluyendo la arqueología, también avanzan notablemente cubriendo aspectos nuevos o profundizando en temas que se venían discutiendo. Ejemplo de ello es el trabajo de José Luís López Ulloa en el terreno histórico, además de trabajos en proceso de Celina Becerra y de Eric Cach en el campo arqueológico. La bibliografía nueva es de tal volumen, que amerita un análisis aparte, lo que sobrepasa los objetivos de este texto. 


\section{Notas sobre el Norte de Jalisco}

La llamada Región Remota por el geógrafo Ángel Bassols Batalla (1988) o Región de los Cañones que decía María Teresa Gutiérrez (1959), es también tierra de rancheros, ganaderos, católicos, al igual que Los Altos de Jalisco. Es también una sociedad variada. Pero contiene un factor que le otorga características diferentes: la presencia de una frontera con el orbe indígena, con el mundo de los Wixárica o Huicholes, que ocupan la parte occidental de la Sierra Madre. Aunado a este factor en el Norte de Jalisco si es posible hablar de una presencia significativa de la Hacienda al lado de las tierras comunales de los indígenas Huicholes y Tepecanos o de los ranchos de mediana y pequeña propiedad. Posteriormente tendrá presencia significativa el ejido. Por cierto, el Norte de Jalisco se localiza en la parte meridional extrema de la Sierra Madre Occidental. Hacia el norte y el oriente, colinda con los amplios valles del centro del estado de Zacatecas y con los de Juchipila y Tlaltenango de la misma entidad. En 1998 iniciamos el trabajo de reconocimiento regional de este territorio bajo el patrocinio de El Colegio de Jalisco, conformándose el llamado «grupo del norte» que llevaría a cabo las pesquisas antropológicas que venían antecedidas por el trabajo, hoy clásico, de Robert Shadow, discípulo de Phil Weigand, en el municipio de Villa Guerrero, a principios de los años 1970 y que se prolongó hasta 1976. Debe mencionarse también el trabajo del historiador David Carbajal López (2002), estudioso de las minas de Bolaños y el de la arqueóloga María Teresa Cabrero, que desarrolló su trabajo a lo largo del río Bolaños. A estos trabajos que he citado como ejemplos, se unen los de María del Cármen Velázquez (1961) y dentro de una nueva generación de investigadores los trabajos de José Rojas o Paulina Ultreras (2005 y 2009). No son los únicos, pero no es mi propósito en este texto analizar la bibliografía existente sobre la región.

Además, en el Norte de Jalisco existe un importante grupo de intelectuales que han contribuido y contribuyen a pensar su realidad regional. Menciono en esta ocasión a Ignacio Bonilla, al finado Manuel Caldera, a Xorge del Campo, Nicolás Valdez, Luís Sandoval Godoy, Fernando Robles Romero, Amador de la Torre Cosío, José Heraclio García y Luís de la Torre, este último, fundador del periódico, hoy suspendido, Mi Pueblo, que constituye una fuente de información imprescindible para la región.

En ese año de 1998, los diez municipios del Norte de Jalisco en su conjunto alcanzaban los 74.035 habitantes, con una densidad media de población de 8.65 habitantes por kilómetro cuadrado, la más baja de Jalisco. Cuando en el año 2000 revisamos el censo, se reportaba para el Norte de Jalisco, un total de 76.172 habitantes, es decir, un crecimiento de sólo 2.137 personas. Más aún, al examinar los datos del censo en 2010, encontramos 78.785 habitantes, es decir, sólo 2.613 más que en el año 2000. La evidencia censal indica que es una tierra despoblada y el trabajo de campo mostró que es una región expulsora de población hacia los Estados Unidos.

Respecto a los índices de marginación, el Norte de Jalisco ocupa los primeros lugares en el estado de Jalisco, con el añadido de que el municipio de Mezquitic es el más extenso del estado y el que presenta los más graves síntomas de sub- desarrollo. Por ejemplo, la intensidad de la pobreza en la Ciudad de Guadalajara era en 2010 de 7.65 mientras que en el norte de Jalisco es de 34.2. En este extremo meridional de la Sierra Madre Occidental en donde se localiza el Norte de Jalisco hay que agregar al Sur Sur-Oeste de Zacatecas que, juntos, conforman la Región de los Cañones. Ese nombre describe con exactitud al terreno de montaña y los frecuentes tajos que lo atraviesan, así como los angostos valles con inclinaciones estrechas y abruptas. Al igual que varias de las regiones de mayor subdesarrollo en América Latina, el Norte de Jalisco dista de ser una tierra pobre en recursos naturales. Entre sus riquezas más notables están las enormes extensiones de pastos en la 
parte del septentrión y en la Sierra Madre Occidental, con sus bosques de coníferas y la fauna asociada. La plata de las minas de Bolaños y el propio cauce de los ríos conforman otra fuente de riqueza natural. Las condiciones de esta Región de los Cañones son propicias para una explotación forestal controlada, la agricultura de temporal y de riego, sobre todo en el propio septentrión y el nororiente. En el norte de la región los terrenos son propicios para la ganadería. Hacia el centro y el sur, la minería extractiva-pero bajo estricta vigilancia-es una opción y hacia el oeste, la explotación forestal y la cría de ganado. El Norte de Jalisco tiene las características para la cría en gran escala del ganado caprino, bovino y porcino. Mención particular merece la situación de la población Wixárica y la importancia de conocer las relaciones entre indígenas y rancheros. El fondo problemático de esa relación es la disputa por la tierra, conflicto que hunde sus raíces desde el período colonial. Las relaciones de trabajo que se pactan entre Huicholes y rancheros son asimétricas, favoreciendo a estos últimos. A ello se agregan los severos problemas que afrontan los Huicholes para comercializar sus productos y su carencia de capital para competir en los mercados regionales. Por supuesto, la sociedad y la cultura Wixárica, llegó a nuestros días con las alteraciones sufridas desde los tiempos coloniales y el permanente contacto con los rancheros y con la sociedad nacional en general. Sin embargo, es una cultura con perfiles particulares que ha conocido la presencia de los investigadores desde tiempos añejos. Citarlos haría la lista muy larga. Como ejemplos menciono los trabajos de Phil Weigand, Jesús Jauregui, José Martínez Torres y Arturo Chamorro, advirtiendo, de nuevo, que no son los únicos.

En la parte oriental del Norte de Jalisco y el Suroeste de Zacatecas, la base social la configuran los rancheros, ganaderos y cultivadores, con relaciones interculturales asimétricas entre la sociedad ranchera y las comunidades Wixaritari. El territorio ocupado por estos últimos en la parte occidental del Norte de Jalisco mide 4.457 kilómetros cuadrados, equivalente al 42\% del total del territorio regional; los rancheros ocupan 5.752 kilómetros cuadrados, es decir, el 58\% del territorio regional. Las relaciones interculturales entre rancheros y wixaritari conforman un entramado que a final de cuentas es parte de la sociedad regional. Pero estas relaciones se hacen desde perspectivas ecológico-culturales muy diferentes. Por ejemplo, los patrones de asentamiento de Huicholes y rancheros difieren notablemente, empezando por la organización de la milpa y la dispersión de los grupos de parentesco que caracteriza a los primeros. Las formas de propiedad son también diferentes. Entre los huicholes cobran nuevamente importancia los grupos de parentesco y las formas comunales de la tenencia de la tierra, mientras que en los rancheros resalta la importancia de las familias nucleares y la propiedad privada.

Los factores históricos señalan las diferencias entre regiones rancheras como la de los Altos de Jalisco y el Norte, aunque ambas forman parte de la expansión castellana hacia la Gran Chichimeca. Las minas del Real de Bolaños constituyeron un importante centro productor de riqueza no solo para la Nueva Galicia sino para la Nueva España en general. La notable arquitectura de la Ciudad de Bolaños, aun con los deterioros del tiempo, expresa la abundancia de recursos que generaron las minas de plata. En un momento, la minería de Bolaños logró sostener una economía regional que también se relacionó con los movimientos de población hacia el Norte, hacia la Gran Chichimeca. El poblado de Chimaltitán, cerca de la ciudad y mineral de Bolaños, está situado en medio de tierras fértiles en donde la agricultura es posible. Tuvo la capacidad de proveer de productos a la ciudad minera y sus alrededores. En este poblado, ocho kilómetros al sur de Bolaños, los franciscanos fundaron en 1616 el Convento de Santiago, que indica también la naturaleza de frontera de estas tierras. Esta interrelación entre ecologías culturales diversas marca otra de las características del Norte de 
Jalisco-y el Sur Sur-Suroeste de Zacatecas- cuya sociedad regional se articuló a través del intercambio de los productos agrícolas, la cría de ganado y la minería.

San Luís Colotlán, la ciudad administrativa regional en el pasado fue sede del Gobierno de las Fronteras (1591), lugar de intensa actividad para pacificar a los chichimecas -con la ayuda de los Tlaxcaltecasy escenario de alguna de las actuaciones del legendario capitán mestizo Miguel Caldera. Con el establecimiento del gobierno de las fronteras se consolidó la presencia militar en la región. Por cierto, José Rojas ha historiado este proceso. Su trabajo aclara varios aspectos de la conflictiva relación entre la Nueva Galicia y la Nueva España. Desde esta última se despachó a los Tlaxcaltecas que fueron parte de los pobladores fundadores de San Luís Colotlán. Precisamente Eugene Sego (1998) ha examinado este proceso. En la fundación de San Luís Colotlán intervino Miguel Caldera, legendario personaje, hijo de una mujer guachichil y un soldado español. El primer Gobernador de San Luís Colotlán, Antonio Gandi, dividió al poblado en barrios siguiendo criterios étnicos. Así, San Luís Colotlán fue una ciudad multicultural habitada por españoles, tlaxcaltecas, guachichiles, afros, purépechas y otomíes. Se configuró un escenario de mestizaje y mezcla cultural notable que incluyó a los propios chichimecas. La actividad militar fue notable a través del Gobierno de las Fronteras y la política de desplazar las mismas hacia más al Norte de la Nueva España. El rancho, la ciudad y el presidio convivieron en esta región que, lejos de ser «remota» en ese momento, fue central desde el punto de vista de las políticas de pacificación y desplazamiento de la frontera aplicada por el Estado Español en sus territorios de ultramar. Los restos de haciendas como San Juan Capistrano (Zacatecas) o San Antonio de Padúa (Jalisco) son testimonio elocuente de la prosperidad regional que logró la articulación de un amplio territorio de frontera. La minería cobró auge en el siglo XVIII, hacia 1747. En 1754, el Real de Minas de Bolaños ascendió a corregimiento como una clara señal de la importancia de la actividad minera no sólo en la articulación regional sino en el contexto de la Nueva España en general. Por cierto, el mineral de Bolaños fue otro motivo de los constantes conflictos entre la Nueva España y la Nueva Galicia. No es sino hasta el reinado de Carlos III que el mineral dicho fue recuperado por Nueva Galicia después de ser expropiado por la Nueva España. La articulación de la minería con la actividad militar del Gobierno de las Fronteras se apuntaló al ser nombrado Antonio de Vivanco como coronel de las milicias de Bolaños al tiempo que era el dueño de las minas. El auge minero de Bolaños terminó en 1848 bajo la propiedad de mineros ingleses. En trabajos como los de David Carvajal se ha demostrado la relación entre la bonanza minera y las oscilaciones demográficas de la ciudad. Más aún, es de suponerse que estos vaivenes poblacionales debieron afectar la consolidación de tradiciones culturales y aún, del arraigo de una identidad local, puesto que ambas debían ser reelaboradas al compás de las oscilaciones provocadas por los altibajos de la minería.

La ganadería y los árboles frutales fueron parte de estrategias adaptativas introducidas por los castellanos. Aún hoy en día es notable la riqueza del banco de plantas descendientes directas de las que plantaron los primeros pobladores castellanos. Sin embargo, el eje estratégico de las nuevas adaptaciones ecológico -culturales fue la ganadería, actividad raíz de la sociedad ranchera, que transformó la toponimia regional con nombres como majadas, vacas-co (devenido en Huacasco), paso del toro, el mular, la estancia, potrero u uncidero. El caballo jugó también un papel de primera importancia. En este contexto, y a diferencia de los Altos de Jalisco, en esta amplia región surgieron grandes haciendas como las ya mencionadas de San Juan Capistrano o San Antonio de Padúa. Pero convivieron con las formas comunales de la propiedad indígena, lo que tendría consecuencias importantes para explicar las relaciones futuras entre la población 
Huichola y Tepecana y el Estado Nacional en momentos muy claves: la aplicación de las Leyes de Reforma y la Guerra Cristera. Estas haciendas fueron en varios casos, como el de Villa Guerrero estudiado por Robert Shadow (2002), el asiento para la formación de poblados y aún de municipios. En estas haciendas ocurrió la transculturación de los pueblos indígenas que vino a unirse a la forma de familias pluriétnicas de poblaciones como Bolaños o la propia San Luís Colotlán. Las haciendas conformaron un rasgo organizativo de la producción que articula a esta región con el amplio Norte de México, la tierra de los grandes latifundios ganaderos. La nueva sociedad emergente en situación de frontera encontró en las haciendas sus mejores apoyos, secundado por el rancho y el presidio, como el de Colotlán.

Una parte de la población chichimeca adoptó la vida sedentaria e influyó en la configuración de nuevas estrategias adaptativas que mezclaron la ganadería con los cultivos, sobre todo, las huertas. En forma paulatina, nómadas y sedentarios establecieron una agricultura adaptada al medio ambiente en convivencia con los ganaderos y mineros. Insisto en la importancia de esta imbricación cultural en la que intervienen los chichimecas (guachichiles, tecuexes, zacatecos) al lado de los tlaxcaltecas, otomíes y purépechas, población de origen africano y españoles. El resultado es una ecología cultural de diversidades, continuada hasta nuestros días, basada en la cría de ganado, la agricultura a campo abierto, la agricultura de huertas, el riego, el cultivo del maguey, el consumo del pulque como ocurre en los poblados de Acaspulco y Acaspulquillo.

Las nuevas adaptaciones introdujeron un variado manejo del agua, no sólo en el aprovechamiento de los ríos, sino en la construcción de aguajes, jagüeyes, bordos, obras de irrigación, que son, entre otros usos, la fuente para el ganado durante el estiaje. Los pozos profundos completaron esta nueva tecnología que intensificó el riego, manejado por españoles y tlaxcaltecas. Así que la sucesión de estrategias adaptativas en el Norte de Jalisco y Sur Sur-Suroeste de Zacatecas siguió una secuencia que combina las prácticas más antiguas de los Chichimecas, basadas en la vida de los cazadores-recolectores, practicantes de una agricultura incipiente, hasta los desarrollos que lograron los habitantes pre hispánicos de las micro regiones de Bolaños y Tépec, para pasar a las introducciones españolas descritas.

La conformación del actual Norte de Jalisco y Sur Sur-Suroeste de Zacatecas consolidó la frontera de la expansión española hacia el noroeste y noreste de México, hacia las profundidades de la Gran Chichimeca, esa dimensión de la historia y la antropología mexicanas que aún plantea un amplio espectro de preguntas por resolver acerca de la formación de la Nación por un lado y del Estado Nacional por el otro.

\section{REFERENCIAS BIBLIOGRÁFICAS}

AGUIRRE BELTRÁN, G. (1986). El quebacer y el pensar de la antropología en México. Puebla: BUAO.

BASSOLS BATALLA, Á. (1988). Norte de Occidente. Una Región Remota de Occidente. México: Instituto de Investigaciones Económicas de la UNAM.

BONFIL BATAlla, G. (1987). 1990, México Profundo. México: CONACUlTA/Grijalbo.

CABRERO MARÍA T. (1989). Civilización en el Norte de México: arqueología de la Cañada del Río Bolaños (Zacatecas y Jalisco), México. Instituto de Investigaciones Antropológicas: UNAM.

CARBAJAL LÓPEZ, D. (2002). La minería en Bolaños. 1784-1810.Ciclos productivos y actores económicos. México: $\mathrm{COLMICH} / \mathrm{U}$ de $\mathrm{G}$.

FÁBREGAS PUIG, A. (1986). La formación histórica de una región: los Altos de Jalisco. México: CIESAS (Col. M.O. de Mendizábal: 5) 
FÁBREGAS PUIG, A. y TOMÉ MARTÍN, P.(2000). Entre Mundos. Procesos Interculturales entre México y España. Zapopan: El Colegio de Jalisco.

FÁBREgAS PUIG, A. (2001).Coord. Memoria del Norte. Coloquio de Colotlán en Homenaje a Manuel Caldera. Zapopan: El Colegio de Jalisco.

FÁBREGAS PUIG, A. (2002). Coord. El Norte de Jalisco. Sociedad, Cultura e Historia en una Región Mexicana. Zapopan: El Colegio de Jalisco.

GONZÁLEZ Y GONZÁLEZ, L. (1968). (1984). Pueblo en Vilo. México: Fondo de Cultura Económica.

GUTIÉRREZ VÁZQUEZ, M. T. (1959). Geografía física de Jalisco. Tesis para obtener el título de licenciado en Geografía. México: Facultad de Filosofía y Letras de la UNAM.

JACKSON TURNER, F. (1890). 1987, «El significado de la frontera en la historia americana», en Secuencia, Número 7, enero-abril, pp. 187-207.

LATTIMORE, O.(1962). «The frontier in History» en Owen Lattimore, Studies in frontier history: Selected Papers. 1928-1958, Oxford: Oxford University Press, 1962.

LÓPEZ ULLOA, J. L. (2013). Entre aromas de incienso y pólvora. Los Altos de Jalisco. México: UIA.

ROJAS GALVÁN, J. (2012). Poder, Cultura y Sociedad. El Gobierno de las Fronteras de San Luis de Colotlán (15911806). México: SEPECH.

SEGO, EUGENE B. (1998). Aliados y adversarios: los colonos tlaxcaltecas en la frontera septentrional de la Nueva España. México: El Colegio de San Luis.

SHADOW, R. D. (2002). Tierra, trabajo y ganado en la región Norte de Jalisco. Una historia agraria de Villa Guerrero, Jalisco (1600-1980). México: COLMICH/U de G.

ULTRERAS VILLAGRANA, P. (2005). La lucha por la tierra: Huejúcar y Tlalcosagua frente a la Hacienda La Quemada, Tesis presentada para obtener el título de Licenciada en Historia. Gudalajara: Universidad de Guadalajara.

ULTRERAS VILLAGRANA, P. (2009). De región de frontera a región de rancheros. Tesis para obtener el grado de Maestra en Ciencias Antropológicas. San Luis Potosí: El Colegio de San Luis.

VELÁZQUEZ, MARÍA DEL C. (1961). Colotlán: doble frontera contra los bárbaros. México: UNAM.

WEIGAND, P. C. (1992). Ensayos sobre el Gran Nayar. Entre Coras, Huicholes y Tepehuanos. México, Centro de Estudios Mexicanos y Centroamericanos de la Embajada de Francia: Instituto Nacional Indigenista.

WEIGAND, P. C. (2002). Coord. Estudio Histórico y Cultural sobre los Huicholes. México: Universidad de Guadalajara.

WOLF, E. (1979). Pueblos y Culturas de Mesoamérica. México: ERA. 